\title{
The kinematics and chemical stratification of the type la supernova remnant 0519-69.0
}

\section{An XMM-Newton and Chandra study}

\author{
D. Kosenko ${ }^{1,2}$, E. A. Helder ${ }^{1}$, and J. Vink ${ }^{1}$ \\ 1 Astronomical Institute Utrecht, Utrecht University, PO Box 80000, 3508TA Utrecht, The Netherlands \\ 2 Sternberg Astronomical Institute, 119992, Universitetski pr., 13, Moscow, Russia \\ e-mail: D.Kosenko@uu.nl
}

Received 18 December 2009 / Accepted 14 March 2010

\section{ABSTRACT}

\begin{abstract}
We present a detailed analysis of the XMM-Newton and Chandra X-ray data of the young type Ia supernova remnant SNR 0519-69.0, which is situated in the Large Magellanic Cloud. We used data from both the Chandra ACIS and XMM-Newton EPIC MOS instruments, and high resolution X-ray spectra obtained with the XMM-Newton reflection grating spectrometer (RGS).

Our analysis of the spatial distribution of X-ray line emission using the Chandra data shows that there is a radial stratification of oxygen, intermediate mass elements (IME) and iron, with the emission from more massive elements peaking more toward the center. Using a deprojection technique we measure a forward shock radius of $4.0 \pm 0.3 \mathrm{pc}$ and a reverse shock radius of $2.7 \pm 0.4 \mathrm{pc}$. We took the observed stratification of the shocked ejecta into account in the modeling of the X-ray spectra, for which we used multicomponent non-equilibrium ionization models, with the components corresponding to layers dominated by one or two elements. An additional component was added in order to represent the shocked interstellar medium, which mostly contributed to the continuum emission. This multicomponent model fits the data adequately, and was also employed to characterize the spectra of distinct regions extracted from the Chandra data. From our spectral analysis we find that the approximate fractional masses of shocked ejecta for the most abundant elements are: $M_{\mathrm{O}} \approx 32 \%, M_{\mathrm{Si} / \mathrm{S}} \approx 7 \% / 5 \%, M_{\mathrm{Ar}+\mathrm{Ca}} \approx 1 \%$ and $M_{\mathrm{Fe}} \approx 55 \%$. From the continuum component we derive a circumstellar density of $n_{\mathrm{H}}=2.4 \pm 0.2 \mathrm{~cm}^{-3}$. This density, together with the measurements of the forward and reverse shock radii suggest an age of 0519-69.0 of $450 \pm 200 \mathrm{yr}$, somewhat lower than, but consistent with the age estimate based on the extent of the light echo $(600 \pm 200 \mathrm{yr})$.

Finally, from the high resolution RGS spectra we measured a Doppler broadening of $\sigma=1873 \pm 50 \mathrm{~km} \mathrm{~s}^{-1}$, from which we derive a forward shock velocity of $v_{\mathrm{FS}}=2770 \pm 500 \mathrm{~km} \mathrm{~s}^{-1}$. We discuss our results in the context of single degenerate explosion models, using semi-analytical and numerical modeling, and compare the characteristics of 0519-69.0 with those of other type Ia supernova remnants.
\end{abstract}

Key words. X-rays: individuals: SNR0519-69.0 - ISM: supernova remnants

\section{Introduction}

Thermonuclear (type Ia) supernova explosions have drawn a lot of attention over the last decade, as they provide a powerful tool to measure cosmological distances (Perlmutter et al. 1999; Riess et al. 1998). The reason is that they are bright enough to be observed over large distances, and that, compared to core collapse supernovae, their peak luminosity shows relatively little variation, which can be further reduced by applying the empirical peak luminosity - decline rate correlation (Phillips et al. 1999). Nevertheless, some evolutionary systematic effects (Panagia 2005) may take place, which may affect the measured values of cosmological parameters. Thus, it is important to understand the mechanism and physics that governs thermonuclear explosions.

In addition to the extensive studies of the supernovae themselves, through their light curves (e.g. Woosley et al. 2007; Blinnikov et al. 2006) and spectra (e.g. Branch et al. 2009), one can also turn the attention to the SN type Ia remnants. A typical young supernova remnant (SNR) is a bright X-ray source, due to the high temperatures of the plasma, heated by the forward and reverse shocks. The reverse shock, propagating inwards into the supernova ejecta, efficiently heats the metal-rich matter. The hot plasma produces X-ray spectra, abound with prominent emission lines. These spectra carry the imprints of the chemical composition of the plasma, and, therefore, the distribution of the elements in the supernova ejecta, which are determined by the explosion properties. Thus, a detailed analysis of the X-ray data of supernova type Ia remnants provides a powerful complementary tool in the studies of the nature of a thermonuclear supernova progenitor (e.g. Sorokina et al. 2004; Badenes et al. 2003).

The current generation of X-ray observatories, such as XMM-Newton and Chandra, provide the capability to obtain simultaneously spectral and imaging data of extended objects such as SNRs. Young SNRs in the Large Magellanic Cloud (LMC) are especially suitable targets for these telescopes, as the relative proximity (48 kpc, e.g. Pietrzyński et al. 2009; Koerwer 2009) of the LMC yields a sufficiently high signal-to-noise level of the X-ray data as observed by the CCDs and grating spectrometers on board Chandra and XMM-Newton. The SNRs are still large enough (typically $\sim 1^{\prime}$ ) to perform morphological studies with Chandra and small enough to be excellent targets for the Reflection Grating Spectrometer of XMM-Newton. An additional advantage is the relatively low interstellar absorption 


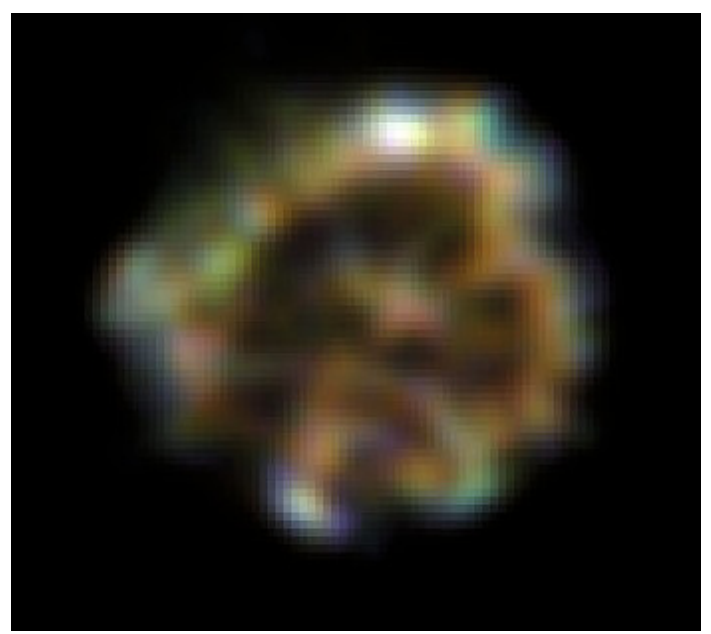

Fig. 1. Chandra smoothed RGB image of SNR 0519-69.0. Red $0.5-1.0 \mathrm{keV}$, green $-1.0-2.0 \mathrm{keV}$, blue $-2.0-4.0 \mathrm{keV}$. North is up, east is left.

column towards LMC SNRs $\left(N_{\mathrm{H}} \approx 10^{21} \mathrm{~cm}^{-2}\right)$, as compared to most Galactic SNRs.

In this paper we present an X-ray study of the LMC SNR 0519-69.0. The SNR has an irregular patchy morphology and an angular extent in X-rays of 33", corresponding to a shell radius of 4 pc. A three color X-ray image of SNR 0519-69.0, based on the Chandra data, is presented in Fig. 1.

In the optical SNR 0519-69.0 has been investigated by Tuohy et al. (1982); Smith et al. (1991) and Ghavamian et al. (2007). It is also one of several LMC SNRs for which a light-echo has been identified, from which an age of $600 \pm 200$ years can be deduced (Rest et al. 2005).

In X-rays the remnant was studied by Hughes et al. (1995), who analyzed the ASCA spectra and found that the SNR is oxygen-poor and iron-rich and must be a remnant of a thermonuclear supernova (SN Ia) explosion. The analysis of Chandra data by Williams et al. (2001) revealed the separation between the shocked ejecta and the shocked circumstellar medium (CSM).

In the present study we analyzed archival X-ray data of SNR 0519-69.0 from the both XMM-Newton and Chandra observatories. We used the ACIS data of Chandra and the imaging spectroscopy (EPIC) and the high spectral resolution grating (RGS) data of XMM-Newton.

Various techniques to analyze the available data are employed. The high spatial resolution Chandra data provide us with an opportunity to study the composition of the SNR as a function of radius. Whereas the RGS data offer high resolution spectroscopy, but without much spatial information.

For the analysis of the SNR 0519-69.0 X-ray spectra, we employed the SPEX (Kaastra et al. 1996) spectral fitting software (version 2.01.05, November 16, 2009). The package contains the most up-to-date atomic data and has a wide range of plasma emission models, which is especially helpful and important in fitting complex spectra from objects such as SNRs.

We fit the XMM-Newton EPIC and RGS spectra with single and multicomponent NEI models. The high spectral resolution RGS data allow us to resolve details of the Fe-L line emission, and to measure line velocity broadening, due to the thermal and bulk motion of the shocked supernova ejecta.

This paper is organized as follows. First in Sect. 2, we describe briefly the Chandra and XMM-Newton data. The methods and techniques we used to deal with the spectral and imaging data are presented in Sect. 3. Section 4 contains the principal results of the study, which are discussed in Sect. 5. We conclude the paper in Sect. 6 .

\section{The data overview}

\subsection{Chandra}

SNR 0519-69.0 was observed only with the ACIS instrument of Chandra X-ray Observatory (obs ID 118) for $41.1 \mathrm{ks}$ on June 21, 2000. We analyzed the data with CIAO 4.1.1 software product and CalDB 4.1.2 calibration data. Apart from an X-ray spectrum of the whole SNR, we also used our own software to extract spectra from certain regions based on emission characteristics. The software reads the standard pipeline event lists and selects events from it based on a "mask" image, i.e. an image containing only zeroes and ones. An event is selected for spectral extraction, if its sky coordinates corresponds with a pixel of value one in the mask image. The event grade selection and spectral data binning scheme are identical to the standard CIAO software. The advantage of this method is that spectra can be extracted from regions with more complex shapes than with the region files, and can be easily constructed based on hardness ratios or narrow band images. These spectra were used together with the ancillary and main response files generated for the SNR as a whole. Given that the remnant occupies only a small part of the ACIS-S3 CCD, using these files for the response is justified. For the background spectrum, we extracted a spectrum from an annulus with radii spanning from $19^{\prime \prime}$ to $37^{\prime \prime}$, centered on the SNR.

\subsection{XMM-Newton}

We used XMM-Newton observations of SNR 0519-69.0 (obs ID 0113000501) that were made on September 17, 2001, and have a total exposure of $47.8 \mathrm{ks}$.

For our study we concentrated on the EPIC MOS and RGS data. Although the EPIC MOS instruments (Turner et al. 2001) have a lower sensitivity than the EPIC pn instrument, they have a higher spectral resolution, which is important for line-rich sources, such as SNRs. The MOS1 observations were performed with thick $(25.4 \mathrm{ks})$ and medium $(15.6 \mathrm{ks})$ filters, whereas the MOS2 data were acquired with the medium filter only. For the spectral analysis the spectra, response and ancillary response files were combined, using weights proportional to the exposure times. For the background, spectra were extracted from an annulus with an inner radius of $2.1^{\prime}$, and an outer radius of $4.3^{\prime}$. This is larger than for the Chandra background spectrum, because the XMM-Newton point spread functions have much broader scattering wings.

The RGS is a slitless spectrometer (den Herder et al. 2001). For an extended source, this implies that the spectrum is smeared by the image of the source itself. For Small/Large Magellanic Cloud remnants, the smearing is modest, but present, and it gives rise to a change in the line spread function. For our analysis, we incorporated this effect into the response matrix by convolving the standard (point source) response matrix with the brightness profile of the SNR, as obtained from the Chandra observations. All the results presented here made use of these modified matrices. This procedure was also applied to the RGS data of the SN 1006 remnant (Vink et al. 2003) and SNR 0509-67.5 (Kosenko et al. 2008).

Apart from adapting the RGS response matrix, all reduction for both MOS and RGS data were made with the standard XMM-Newton software package SAS version 7.1.0. 


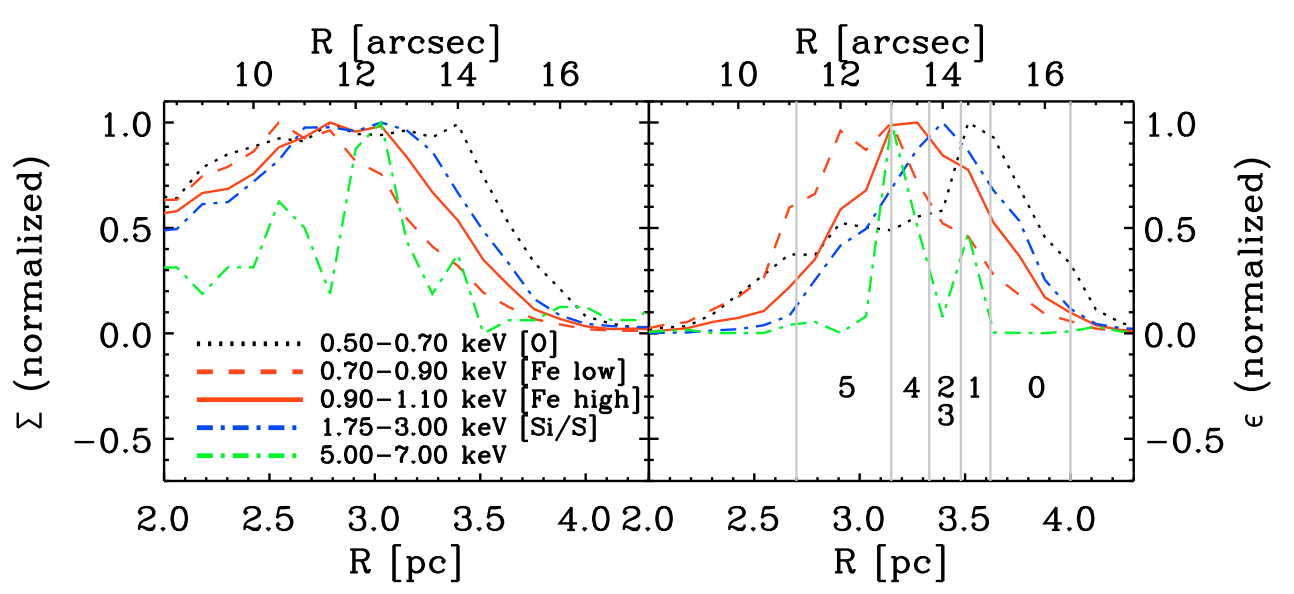

Fig. 2. Azimuthally averaged radial profiles in different energy bands. Left panel: surface brightness radial profiles. Right panel: deprojected emissivity profiles. The vertical light grey lines correspond to the spectral components, used in Sect. 3.4.

Table 1. The inner and outer radii for the SNR 0519-69.0 shell in different energy ranges.

\begin{tabular}{llllllll}
\hline \hline Radii & $0.5-8.0 \mathrm{keV}$ & $\mathrm{CSM}$ & $\mathrm{O}$ & $\mathrm{Si} / \mathrm{S}$ & $\mathrm{Ar} / \mathrm{Ca}$ & Fe-high & Fe-low \\
& & 0 & 1 & 2 & 3 & 4 & 5 \\
\hline$R_{\text {in }}(\mathrm{pc})$ & $2.7 \pm 0.4$ & 3.6 & 3.48 & 3.405 & 3.33 & 3.15 & 2.7 \\
$R_{\text {out }}(\mathrm{pc})$ & $4.0 \pm 0.3$ & 4.0 & 3.6 & 3.48 & 3.405 & 3.33 & 3.15 \\
\hline
\end{tabular}

\section{Data analysis}

\subsection{Chandra radial emissivity profiles}

The high spatial resolution Chandra data allow us to plot radial emissivity profiles of the remnant's shell, with a deprojection technique that was also employed by Helder \& Vink (2008). For the measurements, we first made a radial surface brightness profile of the remnant, using the centroid of the broadband emission $(0.5-8.0 \mathrm{keV})$ as center. Then, assuming that the SNR is spherically symmetric, we used the Lucy-Richardson technique (Lucy 1974; Richardson 1972) to deproject the surface brightness profile into an emissivity profile (following the method of Willingale et al. 1996; Helder \& Vink 2008). We applied this technique to images in different energy bands, where the emission lines from different species dominate. For the oxygen band, we choose $0.5-0.7 \mathrm{keV}$, for the added silicon and sulfur band, we took $1.75-3.00 \mathrm{keV}$. Because the spectra indicated that in the Fe-L band $(\sim 0.7-1.1 \mathrm{keV})$ the relative contribitions from different Fe-L shell ions varies as a function of position (Sect. 3.5.2), we divided the iron energy band in a low $(0.7-0.9 \mathrm{keV})$ and high (0.9-1.1 keV) energy band, which correspond roughly to line emission dominated by Fe XVII and Fe XX ions respectively.

For the broad band $(0.5-8.0 \mathrm{keV})$ energy range, we calculated an inner and outer radius. The inner radius was determined where the emissivity (i.e. deprojected, $\epsilon$ ) drops below one half of the maximum value. We ignored emissivities at radii less than $7^{\prime \prime}$, since the deprojection technique is not well constrained at these lower radii, due to the low contribution to the surface brightness $\Sigma$ of shells at small radii $\left(\Sigma \propto \epsilon(R) R^{2}\right)$. In general, there is no sharp rise in emissivity at the outer radius, so taking a value of one half of the maximum would underestimate the outer radius. Hence, we took a value of $1 / 20$ of the peak value, which, in the deprojections, is just above the noise level of the background at large radii. In the remainder of this paper we assume that this measured inner radius of $2.7 \pm 0.4 \mathrm{pc}$, corresponds to the location of the reverse shock and the outer radius to the forward shock at $4.0 \pm 0.3 \mathrm{pc}$. We estimated the error from the standard deviation of this measurement repeated for 18 individual slices of $20^{\circ}$ each.

We use the deprojected profiles to determine the shells in the remnant in which different species dominate (Fig. 2), which we will use in Sect. 4 for estimating total masses of the shocked species. Table 1 lists the ranges in which the different components dominate. Silicon and sulfur dominate the emissivity in region $2 / 3$, however, our numerical models (see Sect. 5) show that the $\mathrm{Si} / \mathrm{S}$ and $\mathrm{Ar} / \mathrm{Ca}$ layers coincide. For this reason, we split region $2 / 3$ evenly in two parts. Furthermore, we chose $3.6 \mathrm{pc}$ as the division between oxygen and the shocked CSM, since for this value, the volume of the shocked CSM is one fourth of the volume of the total remnant. The latter seems reasonable, if we assume a compression ratio of 4 over the shock front during the whole lifetime of the remnant. Note that all values mentioned above contain uncertainties. For the corresponding volumes, we estimate errors of $50 \%$. In Fig. 2 we also show the observed radial profile and its deprojection for the 5-7 keV hard X-ray band. This band includes both continuum emission and Fe-K shell line emission around $6.5 \mathrm{keV}$. Unfortunately, the signal to noise ratio in this band is poor, but the profile suggests tentatively that the 5-7 keV emission is associated mostly with the Fe XX line emission in the $0.9-1.1 \mathrm{keV}$ band. With some caution, it also suggests that there is no strong non-thermal X-ray continuum associated with the forward shock.

\subsection{EPIC and ACIS spectra}

Fitting the XMM-Newton EPIC MOS and Chandra ACIS spectra with single-ionization timescale non-equillibrium ionization (NEI) SPEX model provides us with the typical values for the emission measure, electron temperature, ionization timescale and abundances in the remnant. The corresponding spectra and the best-fit model are shown in Fig. 3. The best-fit parameters of the spectral model are listed in Table 2. The best-fit abundances are plotted in Fig. 4. For comparison, also the abundances, derived from the analysis of the younger supernova type Ia remnant 0509-67.5 (Kosenko et al. 2008) are plotted. 
A\&A 519, A11 (2010)

Table 2. The best-fit NEI parameters of the XMM-Newton EPIC, RGS and Chandra ACIS data.

\begin{tabular}{lllll}
\hline \hline Parameter & RGS & EPIC MOS + ACIS & ACIS inner & ACIS outer \\
\hline$n_{\mathrm{e}} n_{\mathrm{H}} V\left(10^{58}, \mathrm{~cm}^{-3}\right)$ & $1.8_{-1.8}^{+2.5}$ & $3.7_{-0.2}^{+0.2}$ & $2.63_{-0.32}^{+0.33}$ & $0.66_{-0.08}^{+6.49}$ \\
$k T_{\mathrm{e}}(\mathrm{keV})$ & $2.21_{-0.07}^{+0.08}$ & $2.82_{-0.03}^{+0.03}$ & $2.88_{-0.06}^{+0.06}$ & $1.73_{-1.07}^{+0.14}$ \\
$n_{\mathrm{e}} t\left(10^{10}, \mathrm{~s} \mathrm{~cm}^{-3}\right)$ & $2.69_{-0.03}^{+0.03}$ & $2.27_{-0.01}^{+0.01}$ & $2.22_{-0.002}^{+0.02}$ & $3.90 \pm N / A$ \\
$n_{\mathrm{H}}\left(10^{21}, \mathrm{~cm}^{-2}\right)$ & - & $2.62_{-0.06}^{+0.07}$ & $2.74_{-0.11}^{+0.11}$ & - \\
\hline$\chi^{2} /$ d.o.f. & 2.4 & 29.4 & 10.4 & 2.5 \\
\hline
\end{tabular}

Notes. Columns 1 and 2 list data for the entire remnant. Column 3 lists data for the inner shell of the SNR and Col. 4 - for the outer shell (see Fig. 7).

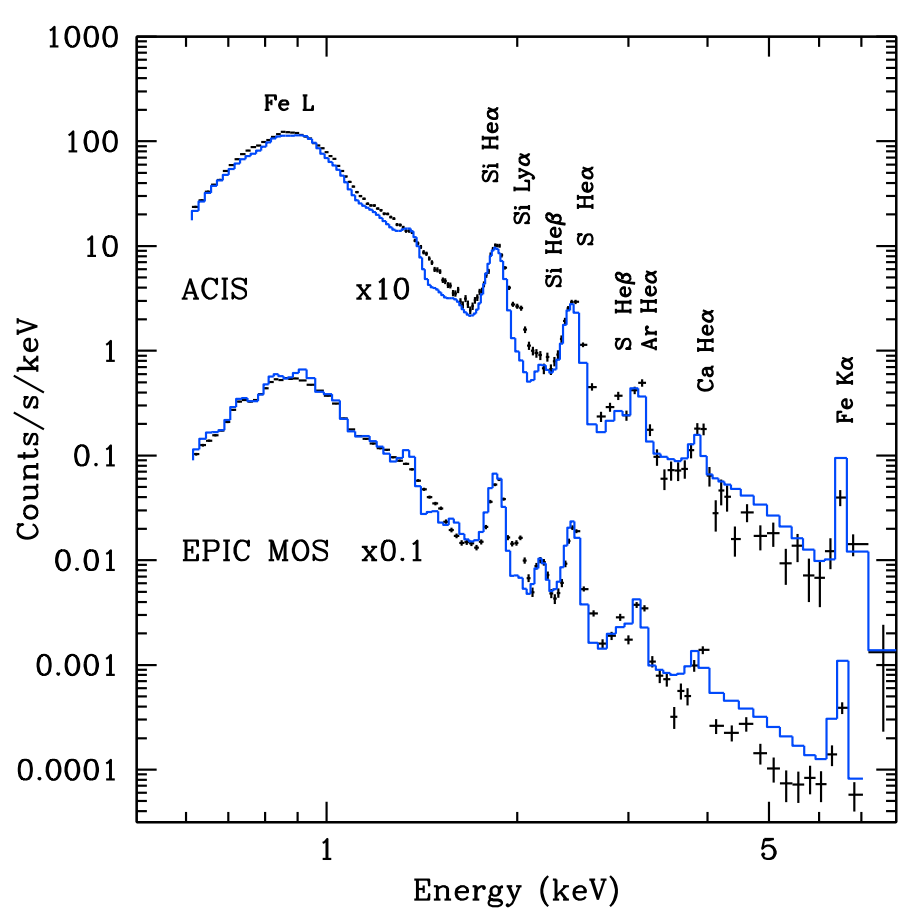

Fig. 3. Combined fitting of the EPIC MOS (scaled down by a factor of 10) and ACIS (scaled up by a factor of 10) data with single ionization timescale NEI model of SPEX.

A single ionization timescale NEI model is not the best approach to explain emission from such a complicated and layered object as a SNR, because in the shell one expects ionization timescale and abundance gradients. For example, Fig. 3 clearly shows that $\mathrm{Si}$ Ly $\alpha$ line is missing in the model and Fe $\mathrm{K}$ line flux is overestimated. This discrepancy leads to a very high value of the fit statistic; $\chi^{2} /$ d.o.f. $\simeq 30$.

Apart from the MOS, we fitted also the EPIC pn data with an NEI model, that yields approximately the same values of the basic parameters of the spectrum.

\subsection{XMM-Newton RGS spectra}

The RGS spectra of SNR 0519-69.0 were also fitted with SPEX NEI models. The fit range was limited to the $0.5-1.1 \mathrm{keV}$ range, which has the more prominent line emission. The best-fit parameters of the single NEI model are listed in Table 2 (first column). This single-ionization timescale model was not able to reproduce the fluxes of O VII and Fe XXI ions: the modeled lines are weaker than the data indicate (top panel of Fig. 5). For this reason, and inspired by the spatial layering, as indicated by the Chandra data (Sect. 3.1), we fitted the RGS spectra in the range of $0.5-1.1 \mathrm{keV}$ with a three-component NEI model: one

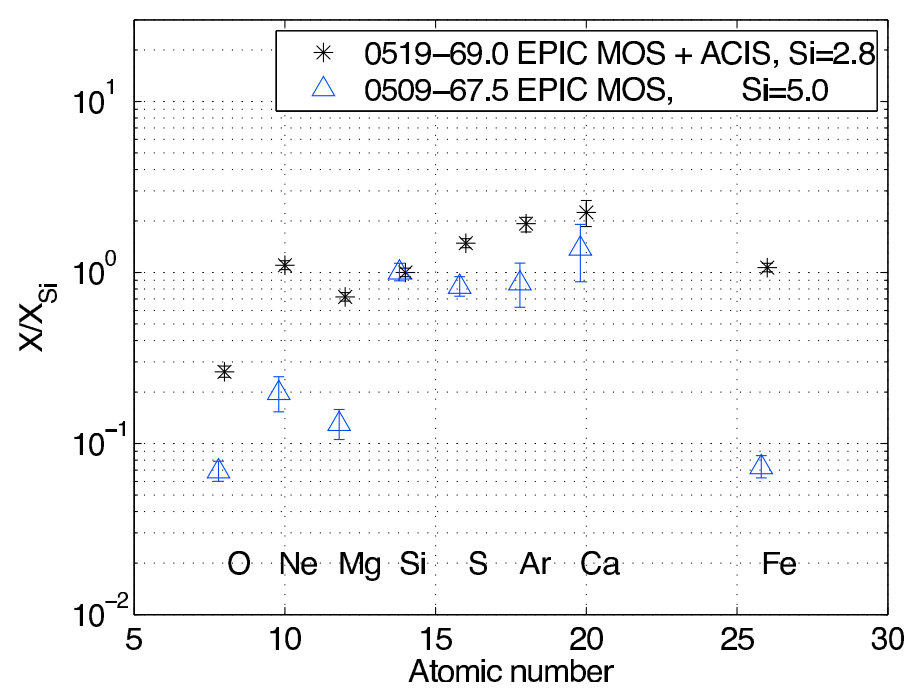

Fig. 4. Best-fit abundances of SNR 0519-69.0 and SNR 0509-67.5 (Kosenko et al. 2008), derived from single-ionization timescale NEI models. The data are in solar units (Anders \& Grevesse 1989), normalized by $\mathrm{Si}$ abundances.

Table 3. The best-fit parameters of the RGS spectral fitting with the three-component NEI model. $\chi^{2} /$ d.o.f. $=1.8$

\begin{tabular}{llll}
\hline \hline Parameter & $\mathrm{O}$ & Fe-low & Fe-high \\
\hline$n_{\mathrm{e}} n_{\mathrm{H}} V\left(10^{58}, \mathrm{~cm}^{-3}\right)$ & $0.50_{-0.06}^{+0.08}$ & $5.6_{-0.3}^{+0.2}$ & $4.8_{-0.7}^{+1.2}$ \\
$k T_{\mathrm{e}}(\mathrm{keV})$ & $0.84_{-0.18}^{+0.27}$ & $1.22_{-0.33}^{+0.05}$ & $2.51_{-0.99}^{+6.54}$ \\
$n_{\mathrm{e}} t\left(10^{10}, \mathrm{~s} \mathrm{~cm}^{-3}\right)$ & $2.3_{-0.5}^{+0.8}$ & $3.0_{-0.2}^{+2.3}$ & $5.2_{-1.4}^{+3.4}$ \\
$\sigma_{\mathrm{RGS}}\left(\mathrm{km} \mathrm{s}^{-1}\right)$ & & $1873 \pm 50$ & \\
\hline
\end{tabular}

component is for pure oxygen, the second - for the low-ionized iron (Fe XVII-Fe XVIII), and the third - for the high-ionized iron (Fe XIX-Fe XXI). The corresponding spectra are shown in the bottom panel of Fig. 5. The corresponding best-fit parameters are listed in Table 3. As can be seen in Fig. 5, this model gives a much better fit to the data than the single NEI model. Note, that no Ne emission is needed to obtain a good fit (Ne IX has a prominent line at $0.92 \mathrm{keV}$ and $\mathrm{Ne} \mathrm{X}$ at $1.02 \mathrm{keV}$ ).

An important advantage of the high spectral resolution RGS spectra is that it enables us to measure the line broadening. The measured best-fit value of the line velocity broadening is $\sigma_{v}=1680 \pm 50 \mathrm{~km} \mathrm{~s}^{-1}$ for the single NEI model and $\sigma_{v}=$ $1873 \pm 50 \mathrm{~km} \mathrm{~s}^{-1}$ for the multi-component model. We adopt the last value as the most reliable value for the Doppler broadening, since the model fits the data much better (Fig. 5). 

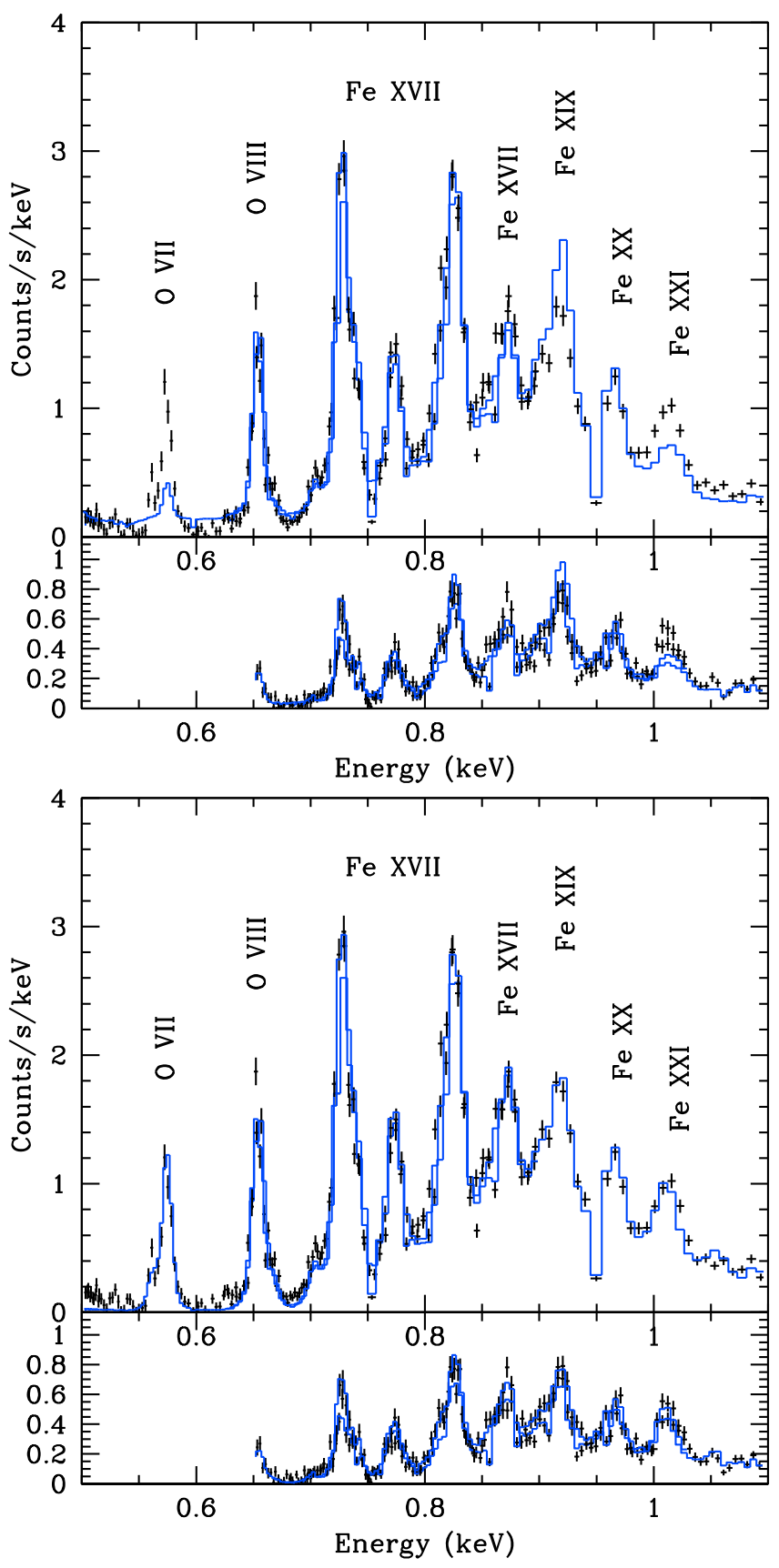

Fig. 5. Top panel: the four RGS spectra RGS1, RGS2 of SNR 051969.0. Shown are both first order (upper frame) and second order (lower frame) spectra and best fit single NEI model including a line velocity broadening model. Bottom panel: the same as the top panel, but here the spectral model consists of three NEI components. Black crosses are the data, solid lines are the models.

\subsection{EPIC MOS and RGS spectra combined, multicomponent approach}

A next step in our study was to combine both the EPIC MOS and RGS data and try to model it with a multi-component NEI model, in which each component roughly corresponds to the layering observed in the radial profiles (Sect. 3.1). In addition to the three components used for fitting the RGS spectra (Sect. 3.3), we added three additional components, which contribute mostly to the emission outside the RGS spectral range. In total this amounts to six components: oxygen (O, or component 1$)$, silicon/sulfur ( $\mathrm{Si} / \mathrm{S}$, or component 2$)$, argon and calcium $(\mathrm{Ar} / \mathrm{Ca}$,

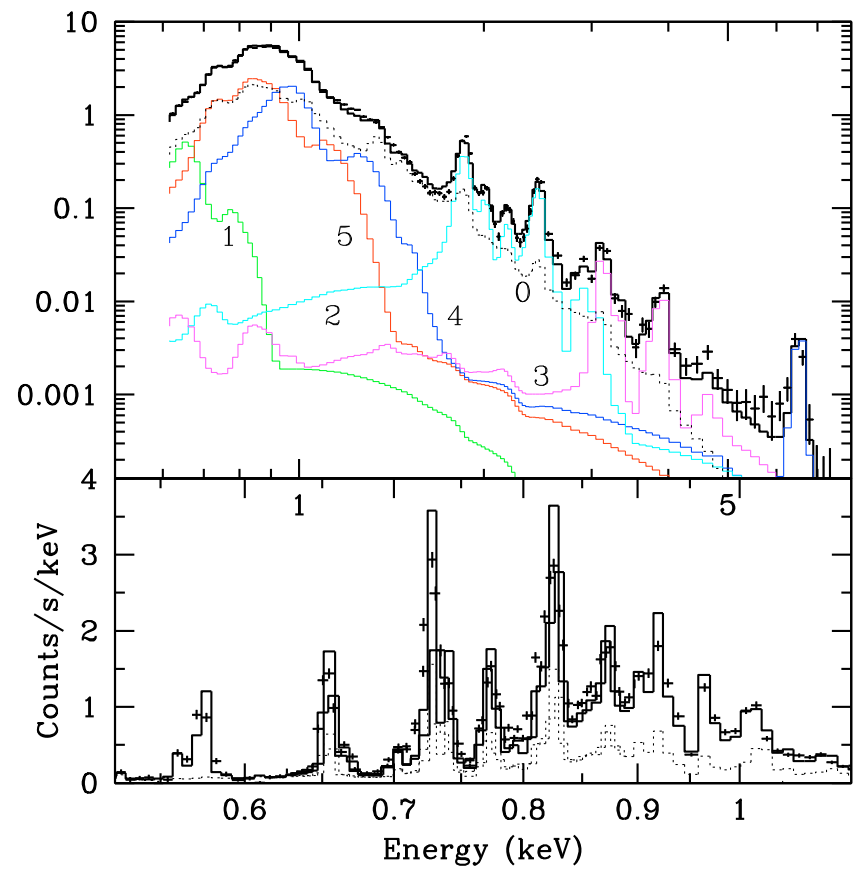

Fig. 6. The EPIC MOS1 and MOS2 combined (upper frame) and the RGS1 and RGS2 first order (lower frame) spectra with the best-fit model of the six NEI components and interstellar absorption. Black crosses are the data, the solid lines show the model. The contributions from the different components are marked with the numbers listed in Table 4. The dotted lines correspond to the CSM component (0).

or component 3), high ionized iron (Fe-high, or component 4), low ionized iron (Fe-low, or component 5), and a continuum dominated model (component 0), for which we used an NEI model with the abundances fixed to those of the LMC (Russell \& Dopita 1992). In addition the model included an interstellar absorption component.

The corresponding principal abundance parameters in components $1-5$ were set to $10^{7}$ times the solar value for the specific elements considered, whereas the abundances of other elements were set to zero. Thereby we secure that the absolute abundances of the corresponding elements are at least two orders of magnitude higher than those of the hydrogen, i.e. for all practical purposes these components correspond to pure metal plasma's. The corresponding spectra and the best-fit multicomponent model are presented in Fig. 6. The best-fit parameters (emission measure, electron temperature, ionization timescale) are listed in Table 4 . The best-fit value for the hydrogen column density is $1.7_{-0.1}^{0.3} \times 10^{21} \mathrm{~cm}^{-2}$.

The multi-component NEI model gives an adequate fit to the data. In contrast to the three NEI component model used to fit only the RGS data, some of the oxygen line emission in this multi-component model attributes part of the corresponding line emission to the shocked CSM component, in particular the O VIII line emission. One peculiarity of the multi-component model is the high $n_{\mathrm{e}} t$ value for $\mathrm{Ar} / \mathrm{Ca}$. This is not an artefact of the model chosen. Isolating that part of the spectrum that is dominated by $\mathrm{Ar} / \mathrm{Ca}$ line emission and searching for other combinations of $k T_{\mathrm{e}}$ and $n_{\mathrm{e}} t$ resulted in similar best fit values. In general, $n_{\mathrm{e}} t$ correlates inversely with $k T_{\mathrm{e}}$. This means that if the $n_{\mathrm{e}} t$ of the plasma is in reality lower than our best fit value, an unrealistically high electron temperature is required. 
Table 4. Parameters of the components in the multi NEI approach. Errors are $1 \sigma \mathrm{rms}, \Delta \chi^{2}=2, \chi^{2} /$ d.o.f. $=7.8$. Emission measure $E M_{\mathrm{X}}=n_{\mathrm{e}} n_{\mathrm{X}} V$.

\begin{tabular}{lllllllll}
\hline \hline Component number & 1 & \multicolumn{2}{c}{2} & & 3 & 4 & 5 & 0 \\
\hline Layer & $\mathrm{O}$ & $\mathrm{Si}$ & $\mathrm{S}$ & $\mathrm{Ar}$ & $\mathrm{Ca}$ & Fe-high & Fe-low & CSM \\
\hline$E M_{\mathrm{X}}\left(10^{54} \mathrm{~cm}^{-3}\right)$ & $8.87_{-0.45}^{+3.39}$ & $2.47_{-0.14}^{+0.31}$ & $1.56_{-0.09}^{+0.20}$ & $0.78_{-0.38}^{+0.63}$ & $0.60_{-0.29}^{+0.49}$ & $2.52_{-0.35}^{+0.32}$ & $2.15_{-0.36}^{+0.17}$ & $2.36_{-0.11}^{+0.50} \times 10^{5}$ \\
$k T_{\mathrm{e}}(\mathrm{keV})$ & $0.72_{-0.24}^{+0.70}$ & $7.00_{-2.26}^{+3.00}$ & $7.00_{-2.26}^{+3.00}$ & $2.67_{-1.17}^{+10.00}$ & $2.67_{-1.17}^{+10.00}$ & $2.73_{-0.27}^{+0.07}$ & $1.26_{-0.26}^{+0.44}$ & $0.64_{-0.06}^{+0.02}$ \\
$n_{\mathrm{e}} t\left(10^{10} \mathrm{~s} \mathrm{~cm}^{3}\right)$ & $1.52_{-0.92}^{+3.66}$ & $3.63_{-0.18}^{+0.27}$ & $3.63_{-0.18}^{+0.27}$ & $27.2_{-18.8}^{+N / A}$ & $27.2_{-18.8}^{+N 1 A}$ & $3.78_{-0.26}^{+0.34}$ & $2.55_{-0.39}^{+0.56}$ & $80.0_{-13.4}^{N / A}$ \\
$n_{\mathrm{e}} / n_{\mathrm{X}}$ & 6.6 & 21.2 & 33.7 & 30.5 & 39.6 & 19.6 & 17.5 & 1.2 \\
\hline
\end{tabular}
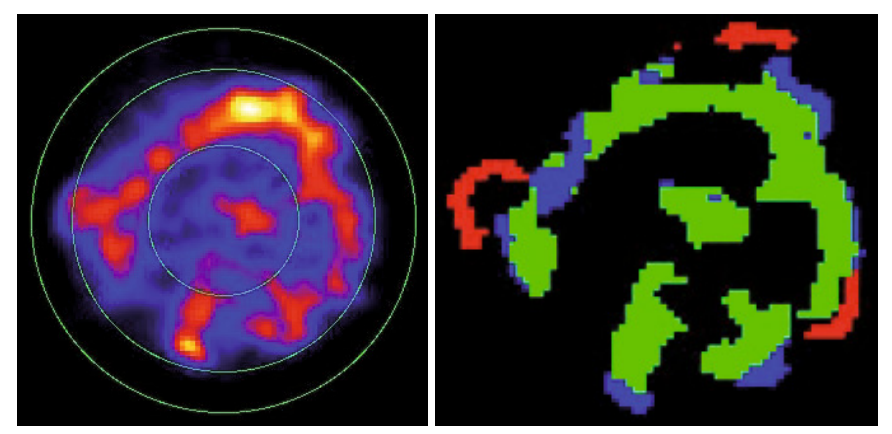

Fig. 7. Left panel: Chandra image of SNR 0519-69.0. Outer and inner regions that were considered are outlined with green lines. Right panel: the masks of the Chandra image of SNR 0519-69.0: red - oxygen $(0.5-0.7 \mathrm{keV})$, green - Fe-low $(0.7-0.9 \mathrm{keV})$, blue - Fe-high $(0.9-1.1 \mathrm{keV})$.

\subsection{Spatially resolved spectroscopy}

\subsubsection{Inner and outer rings of the remnant}

In the analysis of the Chandra data we extracted ACIS spectra from two shells: the outer one which is presumably the shocked CSM of the remnant and the inner one which is the region where the emission is produced by the shocked ejecta material. These regions are outlined with green circles in Fig. 7. Each of the spectra was analyzed in the usual way and fitted with single NEI models. The corresponding best-fit parameters are listed in Table 2.

\subsubsection{ACIS oxygen and iron masks}

The low energy band $(0.5-1.1 \mathrm{keV})$ of the ACIS spectra was treated more thoroughly. We extracted three images of the remnant in the energy bands (see the RGS spectra with the corresponding emission lines) of $0.5-0.7 \mathrm{keV}$ (O only), $0.7-0.9 \mathrm{keV}$ (Fe-low) and 0.9-1.1 keV (Fe-high). Next, three spatial masks were created on the basis of these images, in order to locate regions with the relative emission peaking in either of these energy bands. The masks are presented in Fig. 7. They were applied to the ACIS image of the remnant. Finally, we extracted spectra using these, non-overlapping, image masks.

The resulting spectra are poorly fitted with single-ionization timescale NEI models, the values of the best-fit abundances contain large errors, and physical parameters, such as temperature and ionization timescale are of the order of the typical values obtained in the analysis of the EPIC and ACIS data of the entire remnant. This suggests that the masks did not totally separate out specific layers, but rather that certain layers may dominate the emission in certain regions, with some overlap from other layers. Therefore, we applied the six-component NEI model with the parameters obtained in the fitting of EPIC and RGS data to each of the masked spectra. We fit only emission measures

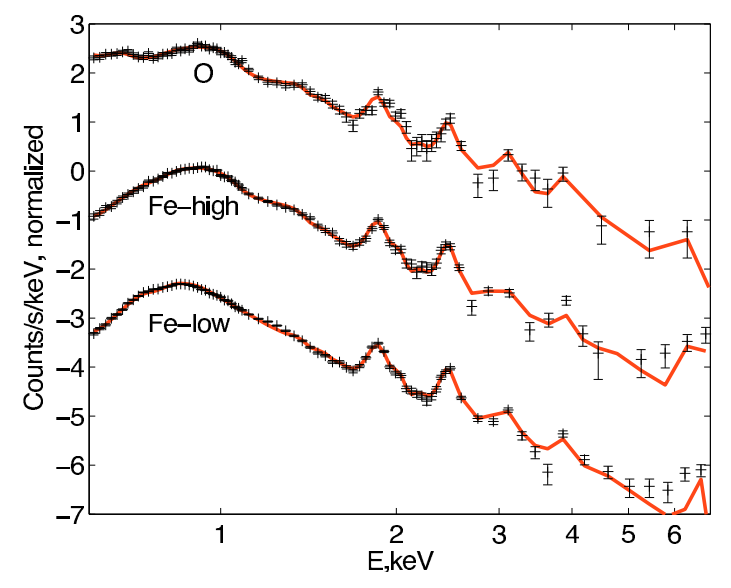

Fig. 8. The three masked ACIS spectra. From top to bottom: oxygen (data shifted upwards for three orders), Fe-high, Fe-low (data shifted downwards for three orders).

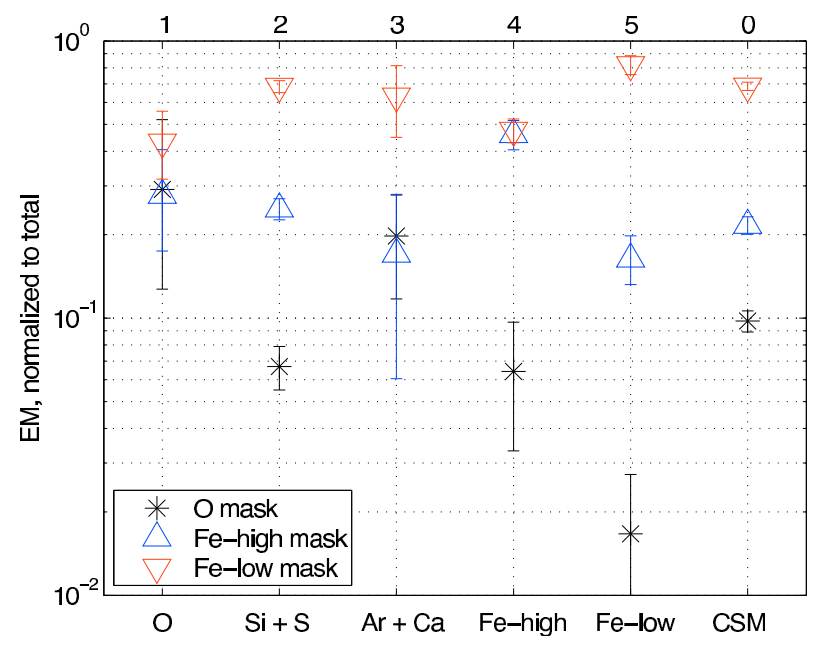

Fig. 9. The emission measure of each of the six NEI components shows the contribution of different elements to the emission of the masked regions of the Chandra data.

(i.e. normalizations) of each component in order to evaluate the contributions of the different components, each corresponding to certain elements, to the spectra of these three distinct regions. The spectra and the best-fit models are presented in Fig. 8.

The contributions of each component to the different spectra are presented in Fig. 9, in which we plot the emission measures of each NEI component.

\section{Results}

The fitting of the XMM-Newton EPIC MOS and Chandra ACIS spectra with SPEX NEI models allowed us to measure (Table 2) the parameters of the plasma, such as typical electron 
temperature $k T_{\mathrm{e}} \simeq 3 \mathrm{keV}$, ionization timescale $n_{\mathrm{e}} t=2.3 \times$ $10^{10} \mathrm{~s} / \mathrm{cm}^{3}$ and abundances (plotted in Fig. 4) in the remnant. The derived best-fit abundances are similar to those of SNR 0509-67.5 (Kosenko et al. 2008), but overall the 0519-69.0 remnant is more metal-rich $(\mathrm{S}, \mathrm{Ar}, \mathrm{Ca}$ and in particular $\mathrm{Fe})$ as it is older and more of the supernova ejecta matter has been swept up by the reverse shock. The relative amount of the light elements (such as $\mathrm{O}, \mathrm{Ne}, \mathrm{Mg}$ ) is comparable for both SNRs.

\subsection{The circumstellar matter density}

The multicomponent NEI model provides us with the parameters of the CSM component (component 0 in Table 4). Using the best-fit emission measure value we can make an estimate of the density of the CSM in the vicinity of the remnant.

$n_{\mathrm{CSM}}=\frac{1}{2} \sqrt{\frac{E M_{\mathrm{LMC}}}{V_{\mathrm{SNR}} n_{\mathrm{e}} / n_{\mathrm{H}}}}=2.4 \pm 0.2 \mathrm{~cm}^{-3}$

where $E M_{\mathrm{LMC}}=2.36_{-0.11}^{+0.50} \times 10^{59} \mathrm{~cm}^{3}$ and $n_{\mathrm{e}} / n_{\mathrm{H}}=1.2$ are taken from Table 4 . We assumed, that the volume of the shocked CSM is $1 / 4 V_{\mathrm{SNR}}$, with $V_{\mathrm{SNR}}=4 \pi / 3 R_{\text {out }}^{3}=8.0 \times 10^{57} \mathrm{~cm}^{3}$ and $R_{\text {out }}=4.0$ pc (Table 1$)$.

\subsection{Structure of the ejecta}

The radial emissivity profiles of the SNR in different energy bands, drawn from the Chandra observations, clearly show stratification of the elements in the remnant. Figure 2 shows that the outermost layer is oxygen rich, the next inward layer produces most of the Si and S emission, all the iron emission comes from the innermost layers of the shell. The iron shell is split in two regions with different ionization properties. The outer layer of high ionized (Fe XX) and the inner one of low ionized (Fe XVII) iron reflect the effects of time-dependent ionization processes in the shocked ejecta: the inner layer has been shocked later by the reverse shock, and therefore has the lowest $n_{\mathrm{e}} t$.

This stratification is also confirmed by the analysis of the masked Chandra images (Fig. 7 right panel) and their spectra (Fig. 8). Figure 9 shows the contribution of different elements to the emission from the masked images.

\subsection{The chemical composition}

The measurements of the inner and outer radii of the shell in different energy bands (Table 1) allow us to estimate the masses of the layers, which have been swept up by the reverse shock. Using the best-fit emission measure value $E M_{\mathrm{X}}=n_{\mathrm{e}} n_{\mathrm{X}} V_{\mathrm{X}}$ of the each model of the multicomponent fitting (Table 4), we can express the mass $M_{\mathrm{X}}$ of an element "X" (i.e. O, Si, S, Ar, Ca, $\mathrm{Fe})$ as

$M_{\mathrm{X}}=n_{\mathrm{X}} V_{X} m_{\mathrm{X}}=\sqrt{\frac{E M_{\mathrm{X}} V_{\mathrm{X}}}{n_{\mathrm{e}} / n_{\mathrm{X}}}} m_{\mathrm{U}} A_{\mathrm{X}}$

where $n_{\mathrm{e}} / n_{\mathrm{X}}-$ electron to ion densities ratio, $m_{\mathrm{U}}-$ atomic mass unit, $A_{\mathrm{X}}-$ atomic mass of the element, $V_{\mathrm{X}}$ - volume occupied by the element (derived from the radii of Table 1).

Combining the available data we obtain $M_{\mathrm{O}}=0.36 M_{\odot}$, $M_{\mathrm{Si} / \mathrm{S}}=0.14 / 0.10 M_{\odot}, M_{\mathrm{Ar} / \mathrm{Ca}}=0.08 / 0.07 M_{\odot}, M_{\mathrm{Fe}}=$ $1.05 M_{\odot}, M_{\text {tot }}=1.8 M_{\odot}$. Note, that the mass values for the elements are affected by the $\sim 50 \%$ errors of the volume estimates, although the total mass is less affected. These masses are higher than may be expected for a single degenerate type Ia
SN model, for which the total mass should be $M_{\mathrm{tot}}=1.4 M_{\odot}$. The most likely reason for this discrepancy may be the errors in the volume estimates or in the filling factor. In particular, inhomogeneities such as knots may give emission measures skewed toward the higher density regions. In fact, the supernova material may be porous and clumpy, thus the emitting volume should be scaled down using a filling factor (e.g. see 3D simulations of thermonuclear explosions by Röpke \& Bruckschen 2008). For example, if we assume volume filling factor in the supernova of $\sim 0.4$, then we get more adequate values $M_{\mathrm{O}}=0.23 M_{\odot}, \quad M_{\mathrm{Si} / \mathrm{S}}=0.09 / 0.07 M_{\odot}, \quad M_{\mathrm{Ar} / \mathrm{Ca}}=$ $0.05 / 0.05 M_{\odot}, M_{\mathrm{Fe}}=0.67 M_{\odot}, M_{\mathrm{tot}}=1.15 M_{\odot}$.

The multicomponent fitting of the XMM-Newton EPIC and RGS spectra reveals a specific behavior of the different NEI components. Table 4 shows that emission measure $\left(E M_{\mathrm{X}}=\right.$ $\left.n_{\mathrm{e}} n_{\mathrm{X}} V_{\mathrm{X}}\right)$ of each component drops from oxygen to calcium, while the values of the ionization timescale parameter $n_{\mathrm{e}} t$ rise from $\mathrm{O}$ to $\mathrm{Ca}$. This trend indicates that increasing from the lighter to the heavier elements, the electron density overcomes the factor of time, which probably does not change drastically for the shocked intermediate mass elements (from $\mathrm{Si}$ to $\mathrm{Ca}$ ). Thus, qualitatively, the decrease of the emission measure and increase of the ionization timescale reflect the relative abundances of the species from $\mathrm{O}$ to $\mathrm{Ca}$, i.e. the quantity $n_{\mathrm{e}} n_{\mathrm{X}} V_{\mathrm{X}} /\left(n_{\mathrm{e}} t\right)=$ $n_{\mathrm{X}} V_{\mathrm{X}} / t \propto M_{\mathrm{X}}$ (total shocked mass of an element $\mathrm{X}$ ) is lower for the heavier species. A similar behavior of the ionization timescale parameter for different species was established by Lewis et al. (2003) in their analysis of the Chandra data of SNR N103B.

From these considerations, applying the time of $t \simeq$ 500 years to all components, we can make rough estimates of the relative contributions of the species in the SNR 0519-69.0 ejecta to be as follows: $M_{\mathrm{O}}=32 \%, M_{\mathrm{Si} / \mathrm{S}}=7 \% / 5 \%, M_{\mathrm{Ar}+\mathrm{Ca}}=$ $1 \%, M_{\mathrm{Fe}}=55 \%$ of the total shocked mass, which are roughly consistent with the mass ratios derived above, using volume estimates. The relative amount of $\mathrm{Ar}$ and $\mathrm{Ca}$ is probably underestimated, due to the high value of ionization timescale parameter measured in the component 3 , for which we do not have a good explanation (note the corresponding errors bars in Table 4).

\section{Discussion}

\subsection{Stratification and composition of the ejecta}

Using the Chandra images we built azimuthally averaged radial emissivity profiles of the SNR in different energy bands, which clearly demonstrate the layering of the elements in the supernova (Fig. 2). Also these profiles reveal the effects of timedependent ionization, as the emission from low ionized iron originates mostly from the inner layers of the iron shell, which is distinct from the high ionized "older" outer iron layers.

These effects are also visible in the Chandra spectra extracted using the masked images (Fig. 7). However, apparently projection effects play a role in that the spectra of the more inward lying layers are contaminated by the outer layers. For example, the spectral fitting with multi-component NEI models shows (Fig. 9) that the oxygen-rich component (component 1) is equally abundant in all three (O, Fe-high, Fe-low) masks. Si and $\mathrm{S}$ (component 2) is almost absent in the O-mask, as it is inside the oxygen-rich layer, but it does contribute to the spectra of inner layers of the Fe-low mask. Ar and $\mathrm{Ca}$ (component 3) tend to be in the outer layers, but the parameters of this component cannot be reliably measured, and have considerable errors. The O-mask 
spectrum hardly contains any contribution from the iron-rich components $(4,5)$, as this outer layer can be isolated without much projection effects.

$\mathrm{Fe}-\mathrm{K}$ emission is present in both the regions corresponding to the Fe-low and Fe-high masks. This is probably due to projection effects, since our spectral analysis suggests that the Fe-K emission is mostly coming from the high ionization layer, which was shocked earlier (component 4, Table 4). The radial emission profiles are consistent with this association, but we note that the signal to noise of the 5-7 keV image on which this is based is low. The high temperature of this high ionization layer may be surprising, but it should be mentioned that the electron temperature depends on both the shock velocity, and the degree of equilibration between electron and ion temperature. In general, one expects that ejecta that is shocked later by the reverse shock is heated to higher temperatures, but this may be off set by the shorter time available for the electrons to equilibrate with the ions. The situation in 0519-69.0 may somewhat resemble the situation in the south of Kepler's SNR. The analysis of Cassam-Chenaï et al. (2004) shows that for this region $\mathrm{Fe}-\mathrm{K}$ peaks at a larger radius than the $\mathrm{Fe}-\mathrm{L}$ emitting region.

\subsection{Comparison of the stratification with other type la supernova remnants}

The chemical stratification of SNR 0519-69.0 is striking. Even more so if one considers that this SNR is much more distant than the well studied Galactic type Ia SNRs Tycho (SN 1572), Kepler (SN 1604) and SN 1006. All these SNRs also show some stratification, but less pronounced. In some cases the $\mathrm{Fe}, \mathrm{Si}$, and $\mathrm{O}$ layers seem to be very close together or even indistinguisable as separate layers. The latter is especially true for Tycho, in which silicon and iron are found very close to the shock front (Warren et al. 2005; Badenes et al. 2006). For Kepler the situation is different for the northern part of the SNR than for the southern part (Cassam-Chenai et al. 2004). In the north the spatial separation between $\mathrm{Si}$ and $\mathrm{Fe}$ is small, whereas in the south the situation is in fact similar to the stratification of SNR 0519-69.0.

The situation for SN 1006 is a bit more complicated. There the O-rich ejecta lie very close to the forward shock (CassamChenaï et al. 2008), but otherwise the Fe-rich layer seems to lie at smaller radius, and may not even have been shocked by the reverse shock, since UV absorption spectra shows the reverse shock to go through Si-rich material (Hamilton et al. 2007).

So what is the origin of this variation in stratification among type Ia SNRs? It could be that there is some variation among type Ia supernovae itself, with some supernovae producing more mixing than others. Optical spectroscopy of type Ia supernovae suggests a strong radial stratification (e.g. Branch et al. 2005; Mazzali et al. 2007) with the the outer ejecta consisting of low mass elements $(\mathrm{O}, \mathrm{Mg})$, then followed by intermediate mass elements (IME), and the inner ejecta consisting of Fe and Ni isotopes. However, a group called High Velocity Gradient (HVG) supernovae (Benetti et al. 2005) show a strong gradient in the $\mathrm{Si}$ II velocities as a function of time, and evidence for high velocity IME. For the HVG SN 2002bo Si appears to be mixed in with O (Stehle et al. 2005). The strong stratification observed in SNR 0519-69.0 suggests, therefore, that its supernova did not belong to the HVG class.

This does not mean, however, that Kepler or Tycho did belong to the HVG class, because other mechanisms may have destroyed the strong layering in the SNR phase. Kepler is in that sense a very interesting case, as the northern and southern regions show different behaviors. In the north the blast wave of Kepler appears to be interacting with a pre-existing shell of about $1 M_{\odot}$, which caused a strong deceleration of the blast wave (Vink 2008). The lack of stratification in that region may therefore be attributed to the strong deceleration, squeezing the different layers closer together.

Interestingly, the expansion rate of Tycho in the eastern part, where the ejecta are very close to the shock front, is also much lower than expected for a SNR expanding in a homogeneous medium (Katsuda et al. 2010). It is, therefore, possible that also in Tycho the initial stratification is no longer visible, as the layers have been squeezed together due to the presence of a strong density gradient, and perhaps even mixed due hydrodynamical instabilities.

Another explanation for the proximity of the ejecta to the shock front in Tycho is the effect of efficient cosmic ray acceleration (Warren et al. 2005). The efficient acceleration alters the equation of state due to the large pressure fraction attributed to relativistic particles and energy losses due to escape of the highest energy cosmic rays (Decourchelle et al. 2000; Helder et al. 2009). The distance between contact discontinuity and shock front is smaller in the east, whereas the X-ray synchrotron rims, a signature of cosmic ray acceleration, are more pronounced in the west. Moreover, one should not confuse the distance between contact discontinuity and shock front, with a lack of stratification within the ejecta. Only if the equation of state within the ejecta is changed, can one explain the lack of stratification by invoking cosmic rays. This could be the case, if also the reverse shock is efficiently accelerating particles. For Tycho there is no evidence that this is the case, but the reverse shock of the core collapse SNR Cas A has been proposed as a site of efficient cosmic ray acceleration (Helder \& Vink 2008).

In addition to cosmic ray acceleration it has been proposed that Rayleigh-Taylor instabilities have brought ejecta close to the shock front (Chevalier et al. 1992; Wang \& Chevalier 2001; Badenes et al. 2006). If this is indeed the case, it may also have destroyed the initial stratification of the ejecta. It is, however, not clear why this mechanism would have affected the stratification of Tycho but not of SNR 0519-69.0, or the southern part of Kepler. So perhaps it is a combination of factors that made Tycho and the north of Kepler less stratified, than 0519-69.0 and the south of Kepler.

\subsection{The chemical composition of SNR 0519-69.0}

From our spectral analysis we derive ejecta masses for $\mathrm{O}, \mathrm{Si} / \mathrm{S}$, $\mathrm{Ar} / \mathrm{Ca}$, and $\mathrm{Fe}$. These masses should be regarded with some caution, because inhomogeneities in the density distribution can lead to some systematic errors. However, on face value, our estimates indicate that the overall abundance pattern matches that for normal type Ia supernovae, but that the oxygen mass is rather large, $M_{\mathrm{O}} \approx 0.2-0.3 M_{\odot}$.

According to a recent analysis of optical spectra of type Ia supernovae (Mazzali et al. 2007) the amount of material from the $\mathrm{C} / \mathrm{O}$ white dwarf burned into IME and more massive elements is remarkably homogeneous, corresponding to a total mass of roughly $M_{\mathrm{IME}, \mathrm{Fe}, \mathrm{Ni}} \approx 1.1 M_{\odot}$. This means that about $0.2-0.3 M_{\odot}$ consists of lower mass elements. This is consistent with the amount we infer for SNR 0519-69.0, provided that most of the outer layers consist of oxygen and that little unburned carbon is present.

One of the differences between pure deflagration and delayed detonation explosion models is that the latter can burn material 
all the way to the outer edge of the white dwarf, leaving no carbon. Optical spectroscopy of type Ia supernovae reveal that little or no carbon is present, with the oxygen abundance a factor 100 to 1000 times the carbon abundance (Marion et al. 2006). The relatively high mass of oxygen in 0519-69.0 is therefore consistent with optical spectroscopy of type Ia supernovae, and supports the idea that burning in the outer layers converts almost all carbon to oxygen.

The amount of Fe that we infer, $\sim 0.7 M_{\odot}$, is also fairly typical for type Ia supernovae. Here we need to caution that not all the Fe may have been shock heated by the reverse shock, so the total iron mass may be higher. Mazzali et al. (2007) showed that the peak luminosity, for which they use the drop in magnitude within 15 days after maximum $\left(\Delta m_{15}\right)$ as a proxy, correlates with the total amount of iron/nickel synthesized (see also Woosley et al. 2007). Using their "Zorro diagram" we can translate our estimate of shocked iron (which includes decayed ${ }^{56} \mathrm{Ni}$ ), in an estimate for $\Delta m_{15} . M_{\mathrm{Fe}}>0.7 M_{\odot}$ corresponds to $\Delta m_{15} \lesssim 1.4$. Combining the amount of IME that we estimate $M_{\mathrm{IME}}=0.2-0.4 M_{\odot}$ with the "Zorro-diagram" suggests that $\Delta m_{15} \gtrsim 1.1$.

These constraints suggest that the supernova was a fairly normal type Ia event, not as bright as $\operatorname{SN1991T}\left(\Delta m_{15}=0.95\right)$, but similar to for example the template type Ia SN $1996 \mathrm{X}\left(\Delta m_{15}=\right.$ 1.3, Salvo et al. 2001). This is unlike the youngest known type Ia SNR in the LMC, 0509-67.5, which was according to the light echo spectrum of the supernova an exceptionally bright type Ia (Rest et al. 2008), similar to SN 1991T.

There is some evidence that bright type Ia supernovae are associated with younger stellar populations (Gallagher et al. 2008), but the starforming history around SNR 0509-67.5 is not significantly younger than around SNR 0519-69.0 (Badenes et al. 2009). This study, therefore, does not provide evidence for a one to one relation between population age and supernova brightness. In addition, Howell et al. (2009) show that the relation between the mean galaxy stellar population age and type Ia brightness has significant scatter. This may be no surprise, if the brightness of a type Ia supernova is related to the properties and history of the progenitor white dwarf, whereas the time delay between birth of the binary system and the supernova event is also governed by the evolution of the secondary star (Howell et al. 2009) ${ }^{1}$.

\subsection{Analytical model}

From the measured locations of the reverse and forward shocks, we can derive typical velocities of the plasma, using the Truelove \& McKee (1999) analytical models, with the ejecta density power $n=7$. Setting the density of the circumstellar matter to $n_{\mathrm{CSM}}=2 \mathrm{~cm}^{-3}$, supernova explosion energy to $E=10^{51} \mathrm{erg}$, and the mass of the progenitor to $M_{\mathrm{WD}}=1.4 M_{\odot}$, we can plot the forward to reverse shock radii ratio versus the time that passed since the explosion. We see from the left panel of Fig. 10 that these parameters correspond to age of the remnant of $460 \pm 150$ years, which is somewhat lower than the value estimated by Rest et al. (2005). The leftmost bottom panel of Fig. 10 shows that in this model, the ratio of the reverse to forward shock velocity is $v_{\mathrm{RS}} / v_{\mathrm{BW}} \simeq 1.2$.

\footnotetext{
${ }_{1}$ Nevertheless, some correlation between time delay and white dwarf properties can be expected, since a long time scale for the evolution of the primary star (the white dwarf that will explode), automatically means that the secondary star evolves on an even longer time scale.
}

\subsection{Numerical HD models}

We run several hydrodynamical models (Sorokina et al. 2004; Kosenko 2006), in order to reproduce the observed features of SNR 0519-69.0. We set the CSM density to $\rho_{\mathrm{CSM}}=$ $3 \times 10^{-24} \mathrm{~g} \mathrm{~cm}^{-3}$. We considered two "toy" explosion models by Woosley et al. (2007) of thermonuclear supernovae: a "mildly mixed" $\operatorname{c050403}\left(M_{\mathrm{Ni}}=0.5 M_{\odot}, M_{\mathrm{Fe}}=\right.$ $0.4 M_{\odot}, M_{\mathrm{IME}}=0.3 M_{\odot}$ ) and a "moderately mixed" m060403 $\left(M_{\mathrm{Ni}}=0.6 M_{\odot}, M_{\mathrm{Fe}}=0.4 M_{\odot}, M_{\mathrm{IME}}=0.3 M_{\odot}\right)$. The distribution of the elements in these models is plotted in the right lower panels of Fig. 10. Velocity and density profiles of the models at the age of 560 years, which are based on these two initial set ups, are presented in Fig. 10 (top graphs).

In the hydrodynamical models the contact discontinuity is further away from the blast wave and closer to the reverse shock as compared to the observed locations in the SNR 0519-69.0. Suggesting that some of the effects that may have brought the ejecta in Tycho's SNR close to the forward shock, whether pure hydrodynamical effects, or efficient cosmic ray acceleration, may to a lesser extent also have affected SNR 0519-69.0. In the near future we intent to produce models that incorporate cosmic ray acceleration into the hydrodynamic models (Kosenko et al. in preparation). Note that Cassam-Chenaï et al. (2008) found that for SN 1006 the contact discontinuity is also close to the shock front, to a lesser extent even for those regions that do not emit X-ray synchrotron radiation, where efficient cosmic ray acceleration is likely not important. Their models suggest that cosmic ray acceleration alone is not sufficient to explain the observations. This suggests that the distance of the contact continuity to the shock front is the result of a mixture of efficient cosmic ray acceleration and hydrodynamical instabilities (see also Miceli et al. 2009; and the discussion in Sect. 5.2).

Estimated from our numerical models, the amount of swept up supernova matter is as follows: $M_{\mathrm{O}}=7 \%, M_{\mathrm{Si} / \mathrm{S}}=$ $12 \% / 7 \%, M_{\mathrm{Ar} / \mathrm{Ca}}=1 \% / 2 \%, M_{\mathrm{Fe}}=63 \%$ for $\mathrm{c} 050403$ and $M_{\mathrm{O}}=3 \%, M_{\mathrm{Si} / \mathrm{S}}=12 \% / 7 \%, M_{\mathrm{Ar} / \mathrm{Ca}}=1 \% / 2 \%, M_{\mathrm{Fe}}=71 \%$ for m060403 explosion model. With a total mass of the shocked ejecta of $1.2 M_{\odot}$ in both cases. We see that the fractional mass of $\mathrm{Fe}$ in $\mathrm{c} 050403$ model matches reasonably well the observed fractional mass as determined in Sect. 4.3, but the observed fractional mass of oxygen appears to be higher than indicated by the models. This may hint that the progenitor was an oxygen-rich white dwarf. The lack of neon in the RGS spectra and magnesium in the EPIC spectra still argues in favor of $\mathrm{C} / \mathrm{O}$ white dwarf, and not an $\mathrm{O} / \mathrm{Ne} / \mathrm{Mg}$ white dwarf, which is consistent with type Ia explosion models (e.g. Woosley et al. 2007).

\subsection{RGS line broadening and the shock velocity}

Using the measured value of the emission lines broadening $\left(\sigma_{\mathrm{RGS}}\right)$, we can estimate the velocity of the forward shock. The temperature of each specie behind the reverse shock is calculated from (no temperature equilibration between species assumed)

$k T_{i}=\frac{3}{16} m_{i} v_{\mathrm{RS}}^{2}$

here $m_{i}-$ mass of species $i, v_{\mathrm{RS}}-$ reverse shock velocity. Thus, the Maxwellian thermal broadening of the lines is

$\sigma_{\text {th ej }}=\sqrt{\frac{k T_{i}}{m_{i}}}=\frac{\sqrt{3}}{4} v_{\text {RS. }}$

As it is shown above, according to the analytical Truelove \& McKee (1999) solution, for the case of SNR 0519-69.0 the 

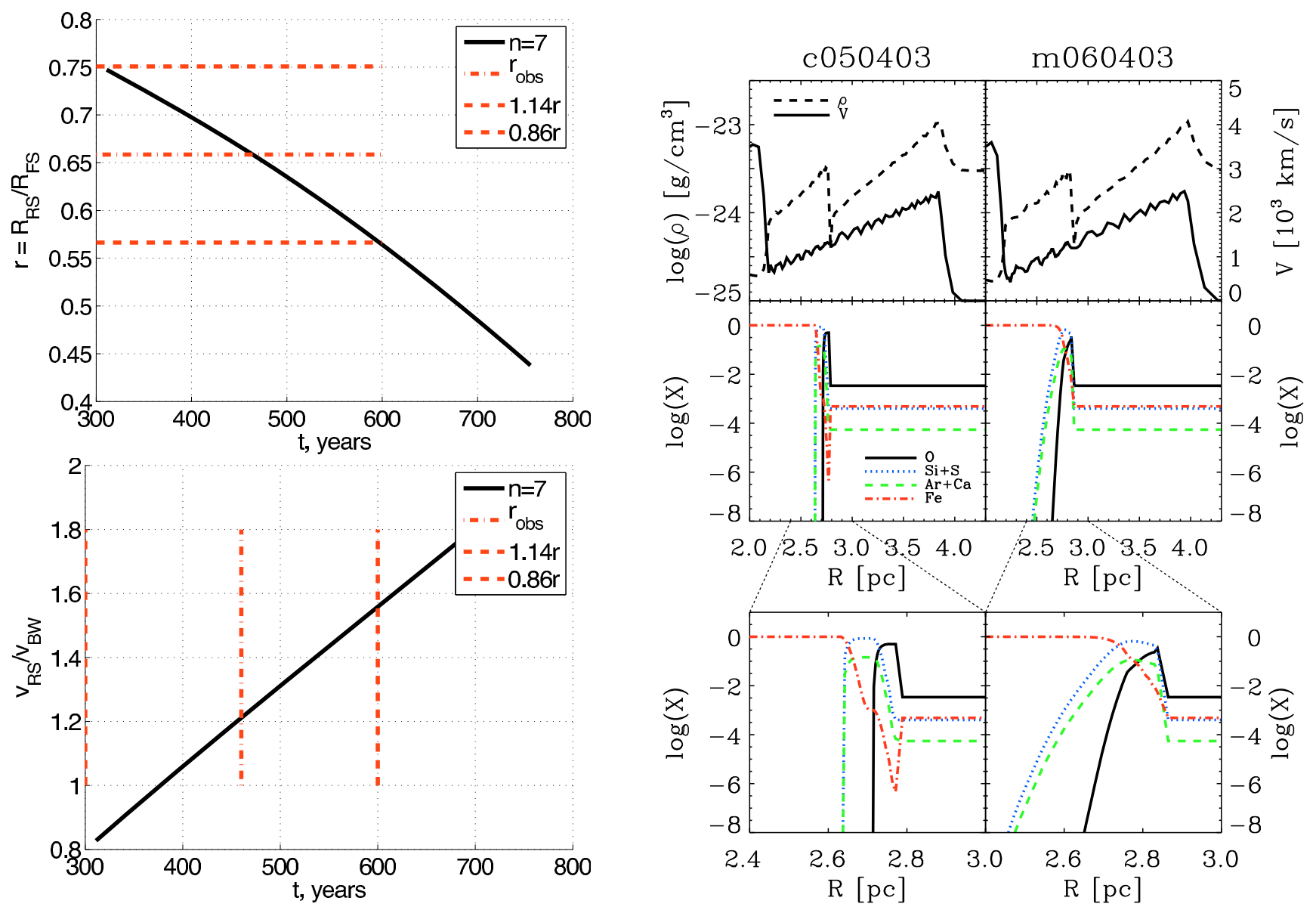

Fig. 10. Left two plots: Truelove \& McKee (1999) analytical solution for SNR 0519-69.0. Radii ratio vs. the age (top panel); red dash-dotted lines show the measured value from the Chandra measurements (Sect. 3.1). Reverse shock velocity (in the frame of the ejecta) to forward shock velocity ratio vs. the age (bottom panel); red dash-dotted lines show values of age derived from the top plot. Right four plots: density, velocity (top panels) and abundances (bottom panels) profiles of the numerical models. In this set, left plots show the "mildly mixed" c050403 model, right plots "moderately mixed" m060403.

relation $v_{\mathrm{RS}}=1.2 v_{\mathrm{FS}}$ holds. Also, according to the numerical models, the value of the bulk velocity in the ejecta is by $\sim 50 \%$ lower than the bulk velocity in the shocked CSM (the velocity profiles of the HD models on the top right plots of Fig. 10), i.e. $\sigma_{\text {bulk ej }} \simeq 0.5 \sigma_{\text {bulk CSM }}=0.5(3 / 4) v_{\text {FS }}$. Thus

$\sigma_{\mathrm{RGS}}=\sqrt{\sigma_{\text {th ej }}^{2}+\sigma_{\text {bulk ej }}^{2}} \simeq 0.64 v_{\mathrm{FS}}$.

Substituting the measured value of $\sigma_{\text {RGS }}=1873 \pm 50 \mathrm{~km} \mathrm{~s}^{-1}$ into Eq. (5), we obtain $v_{\mathrm{FS}}=2927 \pm 500 \mathrm{~km} \mathrm{~s}^{-1}$. Where the error includes an estimate of the systematic uncertainties, which come from relation of $\sigma_{\mathrm{RGS}}$ to $v_{\mathrm{FS}}$.

From the optical observation of the remnant we have independent measurements of the blast wave velocity, but these measurements give ambiguous values. van Adelsberg et al. (2008) modeled hydrogen spectra of the SNR and found shock velocities of $v_{\mathrm{FS}}=2984_{-185}^{+703} \mathrm{~km} \mathrm{~s}^{-1}$ and $v_{\mathrm{FS}}=1178_{-157}^{+185} \mathrm{~km} \mathrm{~s}^{-1}$ based on the observations of Ghavamian et al. (2007) and Smith et al. (1991) respectively. Measurements of $\mathrm{H} \alpha$-line along the rim by Tuohy et al. (1982) provide the value of $v_{\mathrm{FS}}=2800 \pm 300 \mathrm{~km} \mathrm{~s}^{-1}$. Our estimate is the closest to the estimates by Tuohy et al. (1982) and Ghavamian et al. (2007). The lower value of the forward shock velocity in the measurements by Smith et al. (1991) is probably caused by the slit location they used, which intersects the bright eastern rim of the SNR, where the blast wave probably encounters a dense cloud of interstellar matter and may have slowed down (Ghavamian et al. 2007).

\section{Conclusions}

We analyzed X-ray data of SNR 0519-69.0, which were obtained by both the XMM-Newton and Chandra observatories. We treated the spectra with SPEX fitting software and applied nonequiliblium ionization (NEI) models. Fitting of the data with the single-ionization timescale NEI model allowed us to measure average electron temperature of $3 \mathrm{keV}$, ionization timescale of $2.3 \times 10^{10} \mathrm{~s} \mathrm{~cm}^{-3}$.

The stratification of the elements is particularly pronounced in the radial emissivity profiles of the Chandra images of the SNR. Chandra ACIS spectra of specific regions of the SNR also points to the separation of the elements in the shell. This inspired us to use a multi-component fitting model for the EPIC MOS and RGS spectral analysis, in which the observed layers are each represented by an NEI component dominated by one or two elements. This multi-component model provides an adequate description of both the broad band EPIC MOS spectra and the high spectral resolution RGS spectra in the $0.5-1.1 \mathrm{keV}$ band. In addition it allowed us to characterize the Chandra ACIS spectra of several distinct regions. Using this approach we estimate the density of the circumstellar matter of $2.4 \pm 0.2 \mathrm{~cm}^{-3}$. 
The results of our analysis show that the X-ray data of SNR 0519-69.0 are consistent with mildly or moderately mixed delayed detonation explosion models, which result in clear separation of the outermost oxygen layer (produced during the final detonation phase), followed inwards by the partially mixed inner layers of intermediate mass elements and iron (deflagration phase of the explosion). The mass fraction that we infer are $M_{\mathrm{O}} \approx 32 \%, M_{\mathrm{Si} / \mathrm{S}} \approx 7 \% / 5 \%, M_{\mathrm{Ar}+\mathrm{Ca}} \approx 1 \%$ and $M_{\mathrm{Fe}} \approx 55 \%$, which should be considered with some caution, as we had to infer a volume filling factor to convert from emission measure to mass. Taken at face value, these mass fraction are consistent with recent observational results of type Ia supernovae (Mazzali et al. 2007), which show that all material out to an enclosed mass of $\sim 1.1 M_{\odot}$ is burned into IME and heavier elements, leaving about $0.2-0.3 M_{\odot}$ for oxygen, provided all carbon was burned. The mass fractions of IME and Fe in SNR 0519-69.0 puts contraints on the sub-classification of the supernova, suggesting a type Ia event of normal peak luminosity.

The RGS data allowed us to measure the line broadening of the shocked ejecta of $1873 \pm 50 \mathrm{~km} \mathrm{~s}^{-3}$, which helped to constrain the dynamical properties of the remnant and estimate the forward shock velocity of $2770 \pm 500 \mathrm{~km} \mathrm{~s}^{-1}$. This value is consistent with the measurements reported by Tuohy et al. (1982) and Ghavamian et al. (2007), but inconsistent with Smith et al. (1991).

Acknowledgements. We thank the anonymous referee for his/her insightful comments, which helped to broaden the scope of this paper. We also thank S. I. Blinnikov for providing us with the thermonuclear explosion models and also for the fruitful discussions on explosion mechanisms. D.K. is supported by an "open competition" grant and E.H. and J.V. are supported by a Vidi grant from the Netherlands Organization for Scientific Research (PI J. Vink). The work is also partially supported in Russia by RFBR under grant 07-02-00961.

\section{References}

Anders, E., \& Grevesse, N. 1989, Geochim. Cosmochim. Acta, 53, 197

Badenes, C., Bravo, E., Borkowski, K., \& Inmaculada, D. 2003, ApJ, 593, 358

Badenes, C., Borkowski, K. J., Hughes, J. P., Hwang, U., \& Bravo, E. 2006, ApJ, 645, 1373

Badenes, C., Harris, J., Zaritsky, D., \& Prieto, J. L. 2009, ApJ, 700, 727

Benetti, S., Cappellaro, E., Mazzali, P. A., et al. 2005, ApJ, 623, 1011

Blinnikov, S. I., Röpke, F. K., Sorokina, E. I., et al. 2006, A\&A, 453, 229

Branch, D., Baron, E., Hall, N., Melakayil, M., \& Parrent, J. 2005, PASP, 117, 545

Branch, D., Dang, L. C., \& Baron, E. 2009, PASP, 121, 238

Cassam-Chenaï, G., Decourchelle, A., Ballet, J., et al. 2004, A\&A, 414, 545

Cassam-Chenaï, G., Hughes, J. P., Reynoso, E. M., Badenes, C., \& Moffett, D. 2008, ApJ, 680, 1180
Chevalier, R. A., Blondin, J. M., \& Emmering, R. T. 1992, ApJ, 392, 118 Decourchelle, A., Ellison, D., \& Ballet, J. 2000, ApJ, 543, 57

den Herder, J. W., Brinkman, A. C., Kahn, S. M., Branduardi-Raymont, G., \& Thomsen, K. E. A. 2001, A\&A, 365, 7

Gallagher, J. S., Garnavich, P. M., Caldwell, N., et al. 2008, ApJ, 685, 752

Ghavamian, P., Blair, W. P., Sankrit, R., Raymond, J. C., \& Hughes, J. P. 2007, ApJ, 664, 304

Hamilton, A. J. S., Fesen, R. A., \& Blair, W. P. 2007, MNRAS, 381, 771

Helder, E. A., \& Vink, J. 2008, ApJ, 686, 1094

Helder, E. A., Vink, J., Bassa, C. G., et al. 2009, Science, 325, 719

Howell, D. A., Sullivan, M., Brown, E. F., et al. 2009, ApJ, 691, 661

Hughes, J. P., Hayashi, I., Helfand, D., et al. 1995, ApJ, 444, 81

Kaastra, J., Mewe, R., \& Nieuwenhuijzen, H. 1996, in UV and X-ray spectroscopy of astrophysical and laboratory plasmas, ed. K. Yamashita, \& T. Watanabe, 411

Katsuda, S., Petre, R., Hughes, J. P., et al. 2010, ApJ, 709, 1387

Koerwer, J. F. 2009, AJ, 138, 1

Kosenko, D. 2006, MNRAS, 369, 1407

Kosenko, D., Vink, J., Blinnikov, S., \& Rasmussen, A. 2008, A\&A, 490, 223

Lewis, K. T., Burrows, D. N., Hughes, J. P., et al. 2003, ApJ, 582, 770

Lucy, L. B. 1974, AJ, 79, 745

Marion, G. H., Höflich, P., Wheeler, J. C., et al. 2006, ApJ, 645, 1392

Mazzali, P. A., Röpke, F. K., Benetti, S., \& Hillebrandt, W. 2007, Science, 315, 825

Miceli, M., Bocchino, F., Iakubovskyi, D., et al. 2009, A\&A, 501, 239

Panagia, N. 2005, Il Nuovo Cimento B, 120, 667

Perlmutter, S., Aldering, G., Goldhaber, G., et al. 1999, ApJ, 517, 565

Phillips, M. M., Lira, P., Suntzeff, N. B., et al. 1999, AJ, 118, 1766

Pietrzyński, G., Thompson, I. B., Graczyk, D., et al. 2009, ApJ, 697, 862

Rest, A., Suntzeff, N. B., Olsen, K., et al. 2005, Nature, 438, 1132

Rest, A., Matheson, T., Blondin, S., et al. 2008, ApJ, 680, 1137

Richardson, W. H. 1972, J. Opt. Soc. Am., 62, 55

Riess, A. G., Filippenko, A. V., Challis, P., et al. 1998, AJ, 116, 1009

Röpke, F. K., \& Bruckschen, R. 2008, New J. Phys., 10, 125009

Russell, S. C., \& Dopita, M. A. 1992, ApJ, 384, 508

Salvo, M. E., Cappellaro, E., Mazzali, P. A., et al. 2001, MNRAS, 321, 254

Smith, R. C., Kirshner, R. P., Blair, W. P., \& Winkler, P. F. 1991, ApJ, 375, 652

Sorokina, E., Blinnikov, S., Kosenko, D., \& Lundqvist, P. 2004, Astron. Lett., 30,737

Stehle, M., Mazzali, P. A., Benetti, S., \& Hillebrandt, W. 2005, MNRAS, 360, 1231

Truelove, J. K., \& McKee, C. F. 1999, ApJS, 120, 299

Tuohy, I. R., Dopita, M. A., Mathewson, D. S., Long, K. S., \& Helfand, D. J. 1982, ApJ, 261, 473

Turner, M. J. L., Abbey, A., Arnaud, M., Balasini, M., \& Barbera, M. E. A. 2001, A\&A, 365, 27

van Adelsberg, M., Heng, K., McCray, R., \& Raymond, J. C. 2008, ApJ, 689, 1089

Vink, J. 2008, ApJ, 689, 231

Vink, J., Laming, J. M., Gu, M. F., Rasmussen, A., \& Kaastra, J. S. 2003, ApJ, 587, L31

Wang, C.-Y., \& Chevalier, R. A. 2001, ApJ, 549, 1119

Warren, J. S., Hughes, J. P., Badenes, C., et al. 2005, ApJ, 634, 376

Williams, R., Petre, R., \& Holt, S. S. 2001, AIP Conf. Proc., 565, 185

Willingale, R., West, R. G., Pye, J. P., \& Stewart, G. C. 1996, MNRAS, 278, 749

Woosley, S. E., Kasen, D., Blinnikov, S., \& Sorokina, E. 2007, ApJ, 662, 487 